\title{
Early signs of standing postural instability in asymptomatic people living with HIV
}

\author{
Martin G. Rosario \\ Texas Woman's University's School of Physical Therapy, United States
}

\begin{abstract}
Introduction: People with human immunodeficiency virus (HIV) can present vestibular system impairments, affecting postural stability. There is limited literature related to visual and somatosensory systems in maintaining postural stability in HIV-positive people. The purpose of this study was to describe sensory systems used to maintain postural stability and how the sources of sensory information can influence postural stability in asymptomatic persons with HIV.
\end{abstract}

Material and methods: Postural stability was measured in 20 asymptomatic persons with HIV with no history of fall ( 11 males, 9 females; aged $43 \pm 8$ years). Static postural stability was evaluated during eight conditions that perturbed the visual, somatosensory, and vestibular inputs. One-way MANOVA test was conducted to compare center of pressure (COP), antero-posterior displacement (APD), and right-left displacement (RLD) on a stable and unstable surface.

Results: There was a significant increase in the COP, APD, and RLD when the proprioceptive system was challenged on the unstable surface.

Conclusions: Postural instability can be detected in asymptomatic HIV-positive persons with challenging conditions before the evident appearance of balance impairments.

HIV AIDS Rev 2020; 19, 3: 193-198

DOI: https://doi.org/10.5114/hivar.2020.99680

Key words: postural stability, balance, HIV, vestibular, somatosensory, center of pressure.

\section{Introduction}

According to the Centers for Disease Control and Prevention (CDC), human immunodeficiency virus (HIV) is a pathogen that attacks the immune system targeting the CD4 $\mathrm{T}$ cells, reducing their number in the body, and affecting the person's ability to fight infections [1]. One of the HIVs' long-term symptoms can affect the nervous system [2-4]. In addition, studies found that HIV and/or antiretroviral treatment may affect postural stability, increasing fall risk, and affecting quality of life $[2,5,6]$. Furthermore, balance and gait deficits are present in HIV patients, which also lead to an increased fall incidence $[7,8]$.
In order to associate HIV with the nervous system, it is important to recall that structures, such as motor cortex, basal ganglia, and cerebellum, are responsible for controlling and coordinating skeletal muscles as well as posture and balance. Additionally, these structures are in constant communication with the somatosensory cortex and other nervous system areas of sensory control. The systems theory of motor control theory suggests that visual, vestibular, and somatosensory inputs of the nervous system are essential components of sensory processing required to maintain postural stability. This is defined as the ability to maintain the projected center of mass within the limits of the base of support [9]. The description of center of pressure (COP), defined as the vertical projec- 
tion of forces used to control the center of mass, is a method of measuring postural stability [10]. Therefore, while standing quietly, the COP moves around an equilibrium point in the medio-lateral (ML) and anterior-posterior (AP) directions [10].

Subsequently, studies suggest that postural instability in persons with HIV are due to impaired vestibular system $[2,5,11]$. Moreover, magnetic resonance imaging (MRI) studies have demonstrated alterations in the white matter of areas, such as the pontocerebellar tract, which results in gait and postural instability [4]. However, there is a disagreement on the impact of antiretroviral medications and virus stage and the relationship to vestibular impairments in persons with HIV $[2,5,12]$. On the contrary, some authors reported that persons with HIV with vestibular impairments did not report symptoms [2]. Postural instability identified in persons with HIV should be a red flag, as can negatively affect daily activities and quality of life of these individuals [2, 13]. Postural instability can result from alterations in the processing of visual, vestibular, and proprioceptive sensory inputs; for this reason, it is imperative to use postural control tasks focused on particular sensory system [13]. Since there is not much literature available on the involvement of all sensory mechanisms related to postural stability in asymptomatic persons with HIV, it is important to investigate whether vestibular, visual, and somatosensory systems are impaired in this population.

Hence, it is essential to study the integration of sensory systems related to balance in people with HIV, primarily because these persons may be asymptomatic, but with vestibular system disorders and other related complications that can be avoided or treated [2]. Also, it is appropriate to explore which sensory system is impaired the most in relation to balance, in order to establish rehabilitation programs targeting this specific sensory system [13]. In addition, the quality of life of persons with HIV may be affected by balance deficiencies [6], and it is essential to prevent the development of sensory systems impairments to avoid interfering with daily activities [2]. Therefore, the question is: do asymptomatic persons with HIV demonstrate postural instability?

\section{Material and methods}

The institutional review board (IRB) of Texas Woman's University Dallas (protocol No 20092) has reviewed and approved this study before any data collection was initiated. An informed consent was provided to each subject as a requirement to participate in the study and to access the patients' medical records, HIV status, and CD4+ cell count.

\section{Participants}

A total of 34 HIV-positive subjects, aged 25-57 years old, were interviewed and evaluated in the La Perla de Gran Precio (community wellness center that specializes in individuals with HIV) localized in San Juan, Puerto Rico. The in- clusion criteria were: 1 . CD4 levels above 300; 2. Age within the range of 25-57 years; 3 . Walks without an assistive device; 4. Tolerates standing position for at least 30 minutes; 5 . Stable cardiorespiratory system. The exclusion criteria involved: 1. Diagnosis of AIDS (CD4 levels less than 200); 2. Diagnosis of diabetes, dementia, or arthritis; 3 . Severe neuropathy; 4 . Serious balance impairments; 5 . Severe visual acuity problems that are not treated; 6 . BMI $>40$; 7. Decreased functional strength in lower extremities; 8 . Acute proprioception impairments; 9. Falls during the last 6 months; 10. Back or lower extremities lesion or surgery during the last 6 months; 11. Use of medications that causes drowsiness 24 hours prior to intervention; 12 . Women that are pregnant or suspecting pregnancy.

Each subject was evaluated by an interview and evaluation of medical record for the inclusion and exclusion criteria. Various tests were performed as a screening tool to identify additional limitations or impairments in the different sensory systems. To rule out severe neuropathy, Semmes-Weinstein monofilament examination was performed for every participant, with a result of 5.07 or less in more than two areas of the foot $[14,15]$. The visual system was screened using Snellen chart and the subject had to obtain at least 20/40 of visual acuity [16]. To rule out severe balance impairments, Romberg test and Fukuda's stepping test were used. For Romberg test [17], the patients had to maintain standing position for 30 seconds, and Fukuda's stepping test [18] required the subject not rotate more than $30^{\circ}$ from the initial position or deviate more than 0.5 meters from the center of the circle. Functional strength of lower extremities was screened using five-times-to-sit-and-stand test [19], where the subject had to complete the test in 10 seconds or less. Severe proprioception impairments were assessed using proprioceptive awareness test [20], in which the patient was asked to replicate the initial position performed by the evaluator in the contralateral ankle joint in three trials. After screening of the inclusion and exclusion criteria, a total of 20 subjects were able to participate in the study (Table 1, Figure 1).

\section{Procedure}

Each subject was instructed to stand in a static bipedal posture on the MatScan ${ }^{\circledR}$ pressure mat and performed eight balance tasks. Data of center of pressure, antero-posterior sways, and medial-lateral sways were collected in each of the conditions. Each task took 30 seconds to be performed. The first four tasks were performed with the mat on the hard surface of the floor. These four tasks are fully described in Rosario et al. [21].

\section{Data analysis}

Data of postural stability was obtained using the MatScan pressure mat (TekScan Inc., South Boston, MA, USA). Postural stability was operationally defined as the center of pressure displacement, measured by the sensors in 
Table 1. Characteristics of the study participants

\begin{tabular}{l|c}
\hline Characteristics & Study participants $(n=20)$ \\
\hline Age, mean & $42.85 \pm 7.75$ \\
\hline Male, $n$ & $11(55 \%)$ \\
\hline Female, $n$ & $9(45 \%)$ \\
\hline CD4, mean & $607.4 \pm 282.5$ \\
\hline BMI, mean & $27.5 \pm 5.4$ \\
\hline$B M I-$ body mass index
\end{tabular}

the MatScan ${ }^{\circledR}$ pressure mat. These sensors detect the displacement as the body sways anterior, posteriorly, or laterally (right or left). The data collected from the pressure mat was analyzed with Tekscan Sway Analysis Module ${ }^{\mathrm{TM}}$ (SAM) software (TekScan Inc., South Boston, MA, USA) designed for this purpose. Mean and standard deviation were used to describe the data. The statistical software SPSS-25 was used to perform a repeated measure analysis of variance (ANOVA) to compare the center of pressure, antero-posterior, and right-left body sways displacement. $P$-values $<0.0005$ were accepted as statistically significant.

\section{Results}

Our results showed that the magnitude of the center of pressure (COP) was influenced by the standing surface, head movement, and visual input during the eight conditions (Table 2). The purpose of comparing the two surfaces was to detect if asymptomatic persons with HIV present postural instability. First, we compared the stable and unstable surface (Figure 2), dividing the eight conditions according to the surface. For the stable surface conditions group, we used the eyes open condition (EO) as baseline for comparison of COP, antero-posterior displacement (APD), and right-left displacement (RLD). A one-way multivariate analysis of variance (one-way MANOVA) was conducted to compare $\mathrm{EO}$ on the stable surface condition with eyes closed (EC), eyes open + head up and down (EO + HUD), and eyes closed + head up and down (EC + HUD) conditions. There was a significant increase in postural sways when compared with the baseline condition in several of the balance test $F(18,21)=7.0 ; p<0.0005$. Comparing COP and RLD in the different stable surface conditions, no significant increase was found in any of the comparisons. However, APD comparison in different stable surface conditions showed a significant difference between EO $(1.50 \mathrm{~cm})$ and EC + HUD $(2.62 \mathrm{~cm} ; p<0.0005)$.

One-way MANOVA test was performed to compare $\mathrm{EO}$ on the unstable surface condition $(\mathrm{EO}+\mathrm{BF}$ ) (Figure 2) with eyes closed $(\mathrm{EC}+\mathrm{BF})$, eyes open + head up and down $(\mathrm{EO}+\mathrm{HUD}+\mathrm{BF})$, and eyes closed + head up and down $(\mathrm{EC}+\mathrm{HUD}+\mathrm{BF})$ conditions on a foam surface. COP in the unstable surface conditions showed a significant difference between $\mathrm{EO}+\mathrm{BF}\left(7.36 \mathrm{~cm}^{2}\right)$ and $\mathrm{EC}+\mathrm{BF}\left(20.65 \mathrm{~cm}^{2} ; p<0.0005\right)$ and EC + HUD + BF $\left(44.28 \mathrm{~cm}^{2} ; p<0.0005\right)$ when compared.

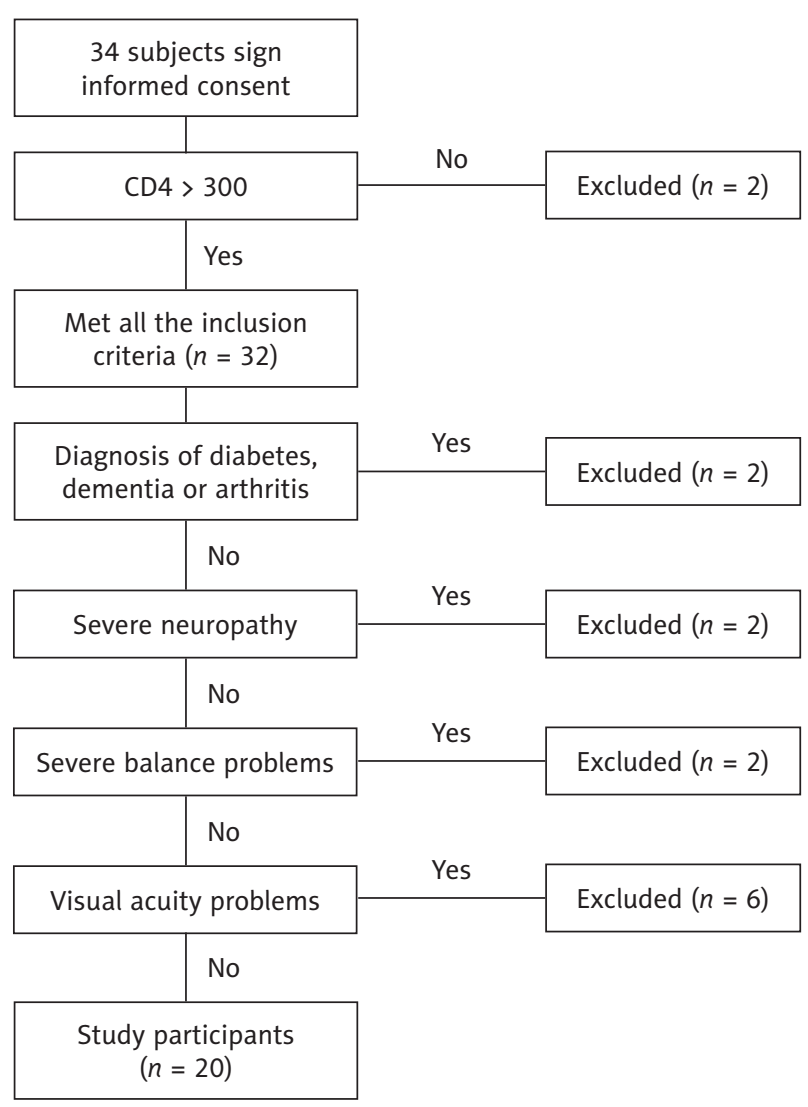

Figure 1. Diagram of subjects excluded and included in the study after screening

In the unstable surface conditions, the APD showed a significant difference between $\mathrm{EO}+\mathrm{BF}(3.81 \mathrm{~cm})$ and $\mathrm{EC}+\mathrm{BF}$ $(6.50 \mathrm{~cm} ; p<0.0005)$ and $\mathrm{EC}+\mathrm{HUD}+\mathrm{BF}(10.78 \mathrm{~cm}$; $p<0.0005)$. In addition, the comparison of RLD in the unstable surface conditions showed a significant difference between $\mathrm{EO}+\mathrm{BF}(3.98 \mathrm{~cm})$ and $\mathrm{EC}+\mathrm{BF}(6.97 \mathrm{~cm} ; p<0.0005)$ and $\mathrm{EC}$ $+\mathrm{HUD}+\mathrm{BF}(9.29 \mathrm{~cm} ; p<0.0005)$.

\section{Discussion}

The objective of the present study was to assess and compare postural stability by altering the sensory systems with changes in surface, head movement, and visual input of asymptomatic subjects with HIV. In the analysis, we compared COP, APD, and RLD in the stable and unstable surface in order to detect postural instability.

For the stable surface, the baseline condition (EO) caused the smallest postural sways in all dimensions compared to other conditions (Table 2). In this condition, there are three sensory systems sending accurate sensory inputs and contributing efficiently to maintaining postural stability [9]. In addition, the APD increased when the vestibular system inputs were altered while standing on the stable surface. To the best of our knowledge, there are no research studies investigated the relationship of the alternation between vestibular inputs 
Table 2. Center of pressure and body sway in the eighth sensory conditions

\begin{tabular}{l|c|c|c}
\hline Condition & COP $\left(\mathrm{cm}^{2}\right)$ & APD $(\mathrm{cm})$ & RLD $(\mathrm{cm})$ \\
\hline EO & $0.756 \pm 0.510$ & $1.499 \pm 0.458$ & $1.013 \pm 0.352$ \\
\hline EC & $1.220 \pm 0.884$ & $2.308 \pm 0.936$ & $1.134 \pm 0.512$ \\
\hline$E O+H U D$ & $1.526 \pm 0.875$ & $2.308 \pm 0.685$ & $1.306 \pm 0.457$ \\
\hline$E C+H U D$ & $1.565 \pm 1.033$ & $2.620 \pm 0.716$ & $1.304 \pm 0.551$ \\
\hline$E O+B F$ & $7.362 \pm 3.993$ & $3.806 \pm 1.274$ & $3.978 \pm 0.976$ \\
\hline$E C+B F$ & $20.649 \pm 10.759$ & $6.505 \pm 1.936$ & $6.975 \pm 2.010$ \\
\hline$E O+H U D+B F$ & $10.629 \pm 3.584$ & $5.200 \pm 1.284$ & $4.707 \pm 1.542$ \\
\hline$E C+H U D+B F$ & $44.284 \pm 28.566$ & $10.779 \pm 3.809$ & $9.293 \pm 3.949$ \\
\hline
\end{tabular}

EO - eyes open, $E C$ - eyes closed, HUD - head up and down, BF - balance foam, COP - center of pressure, RLD - right-left displacement, APD - antero-posterior displacement

A

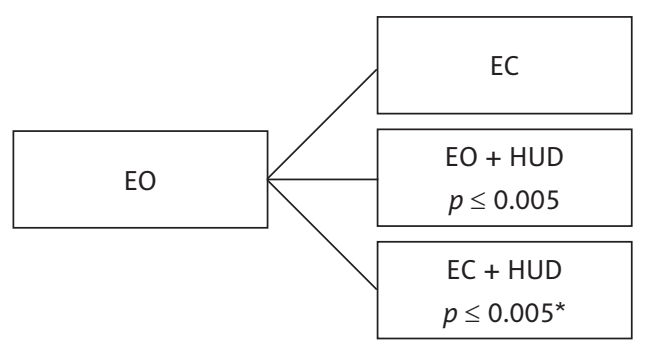

B

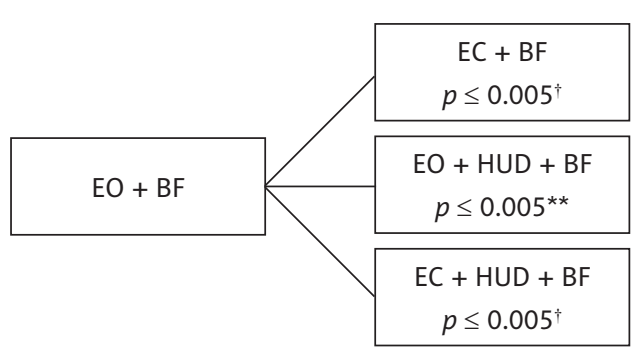

Figure 2. Diagram of comparison within surface. Eyes open condition was established as baseline measurements. A) Stable surface. B) Unstable surface. Measurements: ${ }^{\star} p$-value in APD; ${ }^{* *} p$-value in COP; ${ }^{\dagger} p$-value in COP, APD, and RLD; EO - eyes open, EC - eyes closed, HUD - head up and down, BF - balance foam, RLD - right-left displacement, APD - antero-posterior displacement, COP - center of pressure

and sways in persons with HIV to be compared with our findings.

However, the results of a study with healthy adults reported that head position did not influence the postural sway standing on a firm surface, thus COP and sway could be the same in both conditions [11]. Therefore, our results might suggest some degree of vestibular impairments in persons with HIV (at this point of the disease) $[2,5,6,11]$ that could interfere with the ability of this system to send accurate information to the central nervous system (CNS) to maintain postural stability. Another possible explanation is that persons with HIV could have impairments of vestibulo-ocular reflex (VOR) [22]. The VOR is responsible for stabilizing an image during rapid head movement [22-24]. This mechanism brings accurate information about the body orientation in space to the CNS [23].

Generally, the ankle control strategy (related to APD) is the commonly used approach, compared to the hip control strategy (related to RLD), to control the body sways during quiet stance in stable surface $[9,25]$. The ankle control strategy activates dorsiflexors and plantar flexors, generating small forces that create antero-posterior sways at a slow speed $[9,25]$, and are necessary to control postural stability. In this study, on the stable surface, the APD was approximately $50 \%$ greater than RLD, suggesting that people with
HIV utilized increased ankle strategies to maintain postural control.

The results on the assessment of the unstable surface showed a significant difference between the baseline condition $(\mathrm{EO}+\mathrm{BF})$ compared with $\mathrm{EC}$ and $\mathrm{EC}+\mathrm{HUD}$ $(p<0.0005)$, the remaining unstable surface conditions, indicating postural instability (Figure 2). In this study, there was an increase in the magnitude of COP approximately 6 times the baseline value, when all the sensory systems inputs were altered or eliminated $(\mathrm{EC}+\mathrm{HUD}+\mathrm{BF})$. This test was designed to alter or challenge all sensory input related to balance and postural control. The results showed that among the three balance sensory systems inputs, no accurate information to the CNS was provided resulting in a condition of greater magnitude in COP. Otherwise, in an unstable surface, the magnitude and displacement of COP increased considerably, when the visual input was eliminated.

Trenkwalder et al. [26] found that symptomatic persons with late stages of HIV present difficulties to maintain postural stability with eyes closed on unstable surface. This suggests that, in this study, the visual input had a compensatory role to maintain postural stability when the surface was altered. Authors sustain that visual information is necessary to make postural re-adjustment and maintain postural stability when the somatosensory system inputs are altered [27]. 
Another conclusion of this study was that the values of APD and RLD increased in the unstable surface, resulting in similar values. A possible explanation is that the unstable surface produces faster and larger perturbations that cannot be managed entirely by the ankle control strategy and is necessary to recruit the hip control strategy to control balance [28]. The hip control strategy activates the hip abductors (gluteus medius and tensor fascia latae) and adductor muscle group producing mediolateral sway to maintain postural stability [28]. In these conditions, the ankle and hip control strategies were recruited in a similar way, generating an increase in COP displacement. This could imply that in asymptomatic persons with HIV, the somatosensory system may present deficiencies that produced a great challenge.

For future studies, we suggest measuring COP sway velocity as an outcome of postural stability in people with HIV. Similarly, we suggest collecting information about antiretroviral treatment, since this could be important to determine possible connection between medications and postural instability. Moreover, we advise to assess the integrity of vestibular ocular reflex (VOR) through saccade and smooth pursuit movements. The reason is because VOR is an essential reflex to maintain postural stability in quiet stance, with head movements looking at a fixed point [25].

Therefore, findings of this study reveal that asymptomatic persons with HIV could present postural instability in specific conditions, especially when the proprioception system is challenged. For this reason, it is necessary to promote screening of sensory systems used to maintain postural stability and develop rehabilitation programs directed to prevent and avoid severe postural instability, falls/fall risk, and encourage better quality of life in asymptomatic persons with HIV in early stages of the disease.

\section{Conclusions}

Asymptomatic HIV-positive patients present with postural instability in stable and unstable surfaces. At baseline (EO), participants demonstrated the smallest amount of postural sway. However, as conditions were altered, there was a significant increase in COP, APD, and RLD compared with baseline (EO) indicating that sensory systems could be compromised in asymptomatic persons with HIV. Balance assessments and sensory organization testing should be used to better understand the sensory deficits of the individual. Information gathered in those assessments can help the clinician to design a more comprehensive rehabilitation program focused on restoring postural control and ultimately, improving the balance and safety of asymptomatic persons with HIV.

\section{Acknowledgements}

We would like to thank La Perla de Gran Precio, San Juan, Puerto Rico for providing physical facilities to conduct the study and opportunity to recruit the participants. Ad- ditionally, special thanks to Elizabeth Orozco and Maryvi Gonzalez-Sola for their feedback.

\section{Conflict of interest}

The author declares no conflict of interest with respect to the research, authorship, and/or publication of this article.

\section{References}

1. Definition of HIV. 2017. Available at: https://www.cdc.gov/hiv/basics/whatishiv.html (Accessed: 30.05.2017).

2. Heinze B, Vinck B, Hofmeyr L, Swanepoel D. Vestibular involvement in adults with HIV/AIDS. Auris Nasus Larynx 2014; 41: 160-168.

3. National Institute of Neurological Disorders and Stroke. Neurological complications of AIDS fact Sheet. 2013. Available at: http:// www.ninds.nih.gov/disorders/aids/detail_aids.htm.

4. Sullivan E, Rosenbloom M, Rohlfing T, et al. Pontocerebellar contribution to postural instability and psychomotor slowing in HIV infection without dementia. NIH Public Access 2011; 5: 12-24.

5. Cohen H, Cox C, Springer G, et al. Prevalence of abnormalities in vestibular function and balance among HIV-seronegative women and men. PLoS One 2012; 7: e38419.

6. Heinze B, Swanepoel D, Hofmeyr L. Systematic review of vestibular disorders related to human immunodeficiency virus and acquired immunodeficiency syndrome. J Laryngol Otol 2011; 125: 881-891.

7. Bauer LLO, Ceballos NA, Shanley JJD, et al. Sensorimotor dysfunction in HIV/AIDS: effects of antiretroviral treatment and comorbid psychiatric disorders. AIDS 2005; 19: 495-502.

8. Richert L, Dehail P, Mercie P, et al. High frequency of poor locomotor performance in HIV-infected patients. AIDS 2011; 25: 797-805.

9. Shumway-Cook A, Woollacott M. Normal postural control. In: Motor Control Translating Research into Clinical Practice. $4^{\text {th }}$ ed. Baltimore: Lippincott Williams \& Wilkins; 2012, pp. 162-166.

10. Ruhe A, Fejer R, Walker B. The test-retest reliability of centre of pressure measures in bipedal static task conditions - a systematic review of the literature. Gait Posture 2010; 32: 436-445.

11. Dellepiane M, Medicina MC, Mora R, Salami A. Static and dynamic posturography in patients with asymptomatic HIV-1 infection and AIDS. Acta Otorhinolaryngol Ital 2005; 25: 353-358.

12. Teggi R, Giordano L, Pistorio V, Bussi M. Vestibular function in HIV patients: preliminary report. Acta Otorinolaryngol Ital 2006; 26: 140-146.

13. Adamo D, Pociask F, Goldberg A. The contribution of head position, standing surface and vision to postural control in young adults. J Vestib Res 2013; 23: 33-40.

14. Bell-Krotoski J, Weinstein S, Weinstein C. Testing sensibility, including touch-pressure, two-point discrimination, point localization, and vibration. J Hand Ther 1993; 6: 114-123.

15. Duffy J, Patout C. Management of the insensitive foot in diabetes: lessons learned from Hansen's disease. J Mil Med 1990; 155: 575-579.

16. National Institute of health. Visual acuity test. 2014. Available at: http://www.nlm.nih.gov/medlineplus/spanish/ency/article/003 396.htm

17. Raad J. Rehab Measures: Romberg test. 2014. Available at: http://www. rehabmeasures.org/Lists/RehabMeasures/DispForm.aspx?ID=1173.

18. Fukuda T. The stepping test: two phases of the labyrinthine reflex. Acta Otolaryngol 1959; 50: 95-108.

19. Whitney S, Wrisley D, Marchetti G, et al. Clinical measurement of sit-to-stand performance in people with balance disorders: validity of data for the Five-Times-Sit-to-Stand Test. Phys Ther 2005; 85: 1034-1045.

20. Chui K, Schmitz T. Examination of sensory function. In: O'Sullivan S Schmitz T. Physical Rehabilitation. $5^{\text {th }}$ ed. Philadelphia: F.A. Davis Company; 2007, pp. 87-123. 
21. Rosario MG, Collazo H, Mateo M, Gonzalez-Sola M, Bayron F. Increased static postural sway after energy drink consumption; a randomized trial. F1000 Research 2017; 6: 2036.

22. Heinze BM, Vinck BM, Swanepoel DW. Does the human immunodeficiency virus influence the vestibulocollic reflex pathways? A comparative study. J Laryngol Otol 2014; 128: 772-779.

23. O'Sullivan SB. Examination of motor function: motor control and motor learning. In: O'Sullivan SB. Physical Rehabilitation. $5^{\text {th }}$ ed. Philadelphia, F.A. Davis; 2007, pp. 227-271.

24. Vestibular disorders association. Vision Challenges with Vestibular Disorders. 2009. Available at: http://vestibular.org/sites/default/files/page_files/Vision\%20Challenges.pdf

25. Creath R, Kiemel T, Horak F, Peterka R, Jeka J. A unified view of quiet and perturbed stance: simultaneous co-existing excitable modes. Neurosci Lett 2005; 377: 75-80.

26. Trenkwalder C, Straube A, Paulus W, et al. Postural imbalance: an early sign in HIV-1 infected patients. Eur Arch Psychiatry Clin Neurosci 1992; 241: 267-272.

27. Sarlegna FR, Malfait N, Bringoux L, Bourdin C, Vercher JL. Forcefield adaptation without proprioception: can vision be used to model limb dynamics? Neuropsychologia 2010; 48: 60-67.

28. Cameron M, Monroe L. Balance and fall risk. In: Tyner T, Allen D (eds.). Physical Rehabilitation Evidence-Based Examination, Evaluation, and Intervention. San Louis: Saunders; 2007, pp. 300-332. 